\title{
Evaluación de materiales formativos de acceso público elaborados por bibliotecas universitarias españolas
}

\author{
Marta Somoza-Fernández*, Ernest Abadal*
}

\begin{abstract}
Resumen. Los materiales formativos constituyen recursos muy importantes para la formación de usuarios en bibliotecas. En el estudio se describen y analizan las principales características de los materiales formativos creados por bibliotecas universitarias españolas. Se ha evaluado una muestra de 72 recursos docentes de acceso público aplicando 36 indicadores agrupados en diez categorías. En el análisis se pone de manifiesto que tan sólo el $24 \%$ de las bibliotecas han creado materiales propios y que las temáticas se centran en la explicación de las fuentes de información y los servicios de la biblioteca siendo sólo tres los que abordan la alfabetización informacional. En general, se trata de materiales formativos poco efectivos desde el punto de vista docente (se detecta baja presencia de objetivos formativos, de ejercicios para reforzar el aprendizaje, de pretest, y de evaluación final) y poco interactivos (casi el 50\% son transparencias y el $20 \%$ pdfs). Como conclusión, se muestra que la mayoría de estos materiales se encuentran en una fase inicial de desarrollo y no permiten al usuario realizar de forma completa y autónoma el proceso de aprendizaje.
\end{abstract}

Palabras clave: materiales formativos, tutoriales web, bibliotecas universitarias, evaluación, España.

\section{Analysis of teaching materials created by Spanish academic libraries}

Abstract. Teaching materials are very important for user-training in libraries. This paper describes and analyses the characteristics of the instructional materials created by Spanish academic libraries. It evaluates a sample of 72 resources in public access by applying 36 indicators grouped in ten categories. Only 24\% of libraries were found to have created such materials, focusing on explaining the sources of information and library services. Only three produced materials addressing information literacy. In general, these instructional materials were of poor quality from an educational point of view (few educational objectives, few exercises to reinforce learning, few pre-tests, and little final assessment) and commonly were little interactive (50\% involved slides and $20 \%$ pdfs). In conclusion, most of these teaching materials appeared to be at an early stage of development and did not allow the user to either completely or autonomously follow the learning process.

Keywords: teaching materials, web-based tutorials, academic libraries, evaluation, Spain.

* Facultat de Biblioteconomia i Documentació Universitat de Barcelonan Correo-e: msomoza@ub.edu, abadal@ub.edu

Recibido: 4-3-09; 2. ${ }^{\text {a }}$ versión; 15-5-09; 3. ${ }^{a}$ versión: 5-6-09. 


\section{Antecedentes}

En una encuesta sobre formación de usuarios en bibliotecas universitarias (Somoza, 2007) se ponía de manifiesto un notable interés respecto de la preparación de materiales formativos ya que el $82 \%$ de las bibliotecas elaboraban recursos propios para la formación. Esta misma encuesta recogía además el firme propósito de la mayoría de las bibliotecas universitarias de mejorar estos materiales.

A pesar de este interés manifiesto, en el caso de España, los estudios sobre las características de estos materiales formativos son más bien escasos. Podemos destacar un texto que realiza un análisis global sobre recursos formativos (Sastre, 2000) y el resto son comentarios sobre tutoriales específicos (se trate de MESH, SIRIO o TILT), que se centran en explicar el desarrollo y características de una aplicación en un entorno concreto.

El estudio de Sastre (2000) analiza las guías y tutoriales que difunden 44 bibliotecas universitarias como material didáctico para la formación; en las conclusiones generales sugieren la incorporación de una serie de elementos para mejorarlos (como pueden ser la inclusión de un índice de materias y otro alfabético para facilitar la localización de las bases de datos, entre otros).

En lo que se refiere al apartado de experiencias, uno de los primeros trabajos publicados (Martín, 1994) exponía el proyecto de creación de un tutorial sobre el tesauro MESH de la base de datos MEDLINE, resultado de un proyecto de colaboración entre el Hospital 12 de Octubre y la Universidad Carlos III. Aquí se indicaba que el tutorial debía crearse con Hypercard y también incluir pantallas con los recursos a explicar. Dos años más tarde, Ribes (1996) describe la estructura y contenido de SIRIO (Sistemas de Recuperación de la Información), el primer tutorial elaborado por bibliotecas universitarias españolas, concretamente por la Universidad Politécnica de Valencia. Otro artículo (Seguí, 2001) compara las prestaciones de SIRIO con las de Hot Copy Searching Dialog, dedicado a enseñar el sistema de comandos de Dialog, el conocido distribuidor de bases de datos científicas.

Diversos bibliotecarios de la Universitat Politècnica de Catalunya presentaron el proyecto $A A B I B$ (Gómez, 2002), una plataforma de recursos dedicados a la autoformación y el proyecto FIBU (Formación Inteligente en las Bibliotecas de la UPC) (Méndez, 2002). Estos esfuerzos han culminado con la adaptación del tutorial TILT (Texas Information Literacy Tutorial) y la creación de un tutorial propio para la consulta del catálogo de las Bibliotecas de la UPC.

Somoza $(2002,2005)$ dispone de diversos textos que analizan productos concretos: en el primero se comparan los tutoriales ERIC y PubMed sugiriendo cambios o mejoras siguiendo algunos criterios generales de la alfabetización informacional y en el otro se describe el funcionamiento de programas que permiten la generación de ejercicios (quiz makers), señalando la posibilidad de poderlos hacer parte integrante de tutoriales o también de implementarlos en plataformas formativas. 
Más allá del ámbito universitario, la biblioteca pública de Salamanca «Casa de las Conchas" propone la creación de un tutorial dirigido a usuarios de todas las edades sea cual sea su nivel de formación (Campal, 2006). Este recurso debería estructurarse en cinco partes: los servicios de la biblioteca, la utilización del catálogo, la localización de los materiales, guías de uso de algunas fuentes de información y uso de Internet.

\section{Objetivo y metodología}

El objetivo del presente estudio es evaluar los materiales formativos elaborados por las bibliotecas universitarias españolas y que están en acceso público. Se trata de conocer sus principales características y analizar si incorporan los elementos recomendados en el contexto teórico de la ALFIN. Estos elementos se refieren a tener en cuenta los diferentes niveles de conocimientos que pueden tener los alumnos; a favorecer la autonomía del proceso de aprendizaje del alumno; al establecimiento de opciones de interactividad con el material (no sólo en la interfaz de consulta, sino incluyendo elementos que favorezcan la motivación y el interés por lo que se explica), entre otros aspectos.

Utilizamos el término material formativo para referirnos a los recursos que han sido creados con el objetivo de servir a las actividades de formación de los usuarios en las bibliotecas. Una parte de este material formativo se puede considerar un tutorial cuando cuenta con un conjunto de elementos (ejercicios, sistemas de evaluación, asistencia formativa) que permiten al alumno y también al profesor, si lo hubiera, controlar su proceso de aprendizaje. ${ }^{1}$ A efectos de nuestro estudio distinguimos el material formativo de las guías, que son unos documentos que tienen como propósito fundamental la difusión de los recursos y servicios de la biblioteca y no su aprendizaje. Nuestro análisis, por tanto, se centra en el material formativo (incluyendo los tutoriales) pero dejando al margen aquellos recursos que no tienen objetivos formativos (que hemos denominado "guías").

Para conocer la oferta de títulos no contamos con una base de datos que seleccione y describa el material formativo español como es el caso de PRIMO (Peer Reviewed Instructional Materials Online), elaborado por la ACRL de la ALA, para los tutoriales internacionales. Es por ello que, para llevar a cabo el presente estudio, se han consultado las sedes web de las 72 bibliotecas universitarias españolas.

${ }^{1}$ En el caso concreto de los recursos estudiados sólo cinco cumplirían los requisitos establecidos para un tutorial: TILT (Texas Information Literacy Tutorial) adaptado y traducido por el Servei de Biblioteques i Documentació de la Universitat Politècnica de Catalunya, OPAC búsqueda básica y OPAC búsquedas avanzadas de la Biblioteca de la Universidad de la Laguna, Tutorial en cerca d'informació del CRAI de la Universitat de Barcelona y Búsqueda de información de la Biblioteca de la Universidad de Sevilla. 
Criterios de selección:

- Productor: bibliotecas universitarias; no se han incluido los materiales formativos elaborados y diseñados desde departamentos o facultades ni tampoco aquellos vinculados a empresas relacionadas con la producción o distribución de productos del entorno bibliotecario.

- Temática: sin restricción.

- Plataforma de difusión: web (se han excluido los discos ópticos, por ejemplo).

- Acceso: público (no se han tomado en consideración los productos que están alojados en una intranet o plataforma formativa con acceso restringido).

El análisis se llevó a cabo entre julio y agosto del 2008. Se consultaron las páginas web de todas las bibliotecas universitarias españolas seleccionándose un total de 72 productos (Anexo).

A partir de esta selección se ha elaborado una base de datos sistematizando los principales indicadores y describiendo las principales características referidas a los contenidos, la asistencia formativa, la navegación y diseño y finalmente las de carácter tecnológico.

Existen estudios similares a la metodología propuesta en el presente estudio con el fin de evaluar el material formativo. Un reciente artículo (Marzal, 2008) establece un modelo evaluativo de la usabilidad de los materiales formativos virtuales. Los criterios han sido extraídos a partir de las aportaciones teóricas del campo de la cognición humana, del conocimiento y las teorías pedagógicas y de la usabilidad, presentando un total de veintitrés indicadores agrupados en tres categorías: adaptación centrada en la atención, fidelización vinculada a la percepción y capacidad alfabetizadora relacionada con la memoria. El modelo propuesto fue validado en la práctica por 364 alumnos de primaria y secundaria evaluando dos recursos educativos.

En el caso del presente estudio, para llevar a cabo la evaluación sistemática de los recursos seleccionados, se han establecido un conjunto de 35 indicadores (recogidos en la Tabla I) que se agrupan en diez grandes apartados: aspectos generales, contenidos, aspectos docentes, evaluación del alumno, asistencia, evaluación del material formativo, autonomía del proceso de aprendizaje, alumnos con conocimientos diferentes, navegación, diseño e interactividad, y aspectos tecnológicos. Estos indicadores constituyen una propuesta propia que se basan en la bibliografía sobre evaluación de material didáctico (Richadeau, 1986 y Parcerisa, 2001) que tiene en cuenta la fase de planificación de la actividad, su desarrollo y los resultados, y también en los estudios sobre usabilidad en general (Nielsen, 2000, 2006). Han sido ya usados en un artículo anterior sobre evaluación de tutoriales web internacionales (Somoza; Abadal, 2007). 


\section{TABLA I}

\section{Indicadores seleccionados}

\begin{tabular}{|c|c|c|}
\hline & Indicador & Descripción \\
\hline \multirow{5}{*}{$\begin{array}{l}\text { Aspectos } \\
\text { generales }\end{array}$} & Idioma & \\
\hline & Fecha de creación & \\
\hline & Fecha actualización & \\
\hline & Background & Explica el proceso de creación \\
\hline & Autoría & $\begin{array}{l}\text { Especifica las personas responsables de la crea- } \\
\text { ción del material formativo }\end{array}$ \\
\hline \multirow{3}{*}{ Contenidos } & Tipología & Textual, imágenes, etc. \\
\hline & Estructuración & Organización de los contenidos (modular o lineal) \\
\hline & Temáticas & Materias tratadas por el material formativo \\
\hline \multirow{4}{*}{$\begin{array}{l}\text { Aspectos } \\
\text { docentes }\end{array}$} & Ejemplos & $\begin{array}{l}\text { Presencia de ejemplos que vinculan la teoría con } \\
\text { la práctica }\end{array}$ \\
\hline & Presencia de glosario & \\
\hline & Enlaces externos & Recursos que complementan los contenidos \\
\hline & Metodología docente & $\begin{array}{l}\text { Exposición de contenidos, demostración guiada, } \\
\text { resolución de problemas }\end{array}$ \\
\hline \multirow{2}{*}{$\begin{array}{l}\text { Evaluación del } \\
\text { alumno }\end{array}$} & $\begin{array}{l}\text { Presencia y tipología de } \\
\text { ejercicios }\end{array}$ & Cuestionario, prácticas, juegos, etc. \\
\hline & $\begin{array}{l}\text { Valoración de los ejer- } \\
\text { cicios }\end{array}$ & $\begin{array}{l}\text { Existe alguna forma de evaluación o de feedback } \\
\text { de los ejercicios }\end{array}$ \\
\hline Asistencia & $\begin{array}{l}\text { Contacto con el biblio- } \\
\text { tecario }\end{array}$ & $\begin{array}{l}\text { De esta forma se pueden plantear dudas o co- } \\
\text { mentarios }\end{array}$ \\
\hline $\begin{array}{l}\text { Evaluación del } \\
\text { material } \\
\text { formativo }\end{array}$ & $\begin{array}{l}\text { Sistema de evaluación } \\
\text { del material formativo }\end{array}$ & $\begin{array}{l}\text { Se analiza si el material formativo permite que } \\
\text { el usuario opine sobre los contenidos y sugiera } \\
\text { mejoras. }\end{array}$ \\
\hline \multirow{4}{*}{$\begin{array}{l}\text { Autonomía del } \\
\text { proceso de } \\
\text { aprendizaje }\end{array}$} & Indicación de objetivos & Presentación de objetivos formativos \\
\hline & Pretest & $\begin{array}{l}\text { Inclusión de algún sistema para evaluar los co- } \\
\text { nocimientos previos }\end{array}$ \\
\hline & Temporalización & $\begin{array}{l}\text { Indicación del tiempo estimado para cursar cada } \\
\text { uno de los apartados. }\end{array}$ \\
\hline & Presencia de sumario & $\begin{array}{l}\text { Resumen de los principales contenidos al final } \\
\text { de cada módulo }\end{array}$ \\
\hline \multirow{3}{*}{$\begin{array}{l}\text { Alumnos } \\
\text { con conocimien- } \\
\text { tos diferentes }\end{array}$} & Niveles & $\begin{array}{l}\text { Posibilidad de adaptarse a distintos niveles de } \\
\text { conocimiento por parte del usuario (general, } \\
\text { avanzado, etc.). }\end{array}$ \\
\hline & Perfil de usuario & Tipología de usuario al que va destinado \\
\hline & Formación a distancia & $\begin{array}{l}\text { Preparación de los contenidos para contemplar } \\
\text { esta tipología concreta de usuario }\end{array}$ \\
\hline
\end{tabular}




\begin{tabular}{|c|c|c|}
\hline & Indicador & Descripción \\
\hline \multirow{8}{*}{$\begin{array}{l}\text { Navegación y } \\
\text { diseño }\end{array}$} & Guía de navegación & \\
\hline & Mapa del sitio & \\
\hline & Barra de situación & \\
\hline & $\begin{array}{l}\text { Personalización de la } \\
\text { pantalla y colores }\end{array}$ & \\
\hline & $\begin{array}{l}\text { Cambio tamaño letra o } \\
\text { contraste de la pantalla }\end{array}$ & \\
\hline & Animaciones & Facilitan la interactividad con el sistema \\
\hline & Elementos multimedia & Utilización de audio y vídeo. \\
\hline & Contextos didácticos & $\begin{array}{l}\text { Diferentes escenarios que hacen que el materia } \\
\text { formativo sea menos formal y facilite la interac- } \\
\text { ción con los contenidos }\end{array}$ \\
\hline \multirow{4}{*}{ Tecnología } & $\begin{array}{l}\text { Necesidad de código } \\
\text { de entrada }\end{array}$ & \\
\hline & Descarga plugins & \\
\hline & Distintas versiones & $\begin{array}{l}\text { Posibilidad de consultar el material formativo en } \\
\text { distintas versiones ( } \sin \text { animación, texto básico } \\
\text { etc.). }\end{array}$ \\
\hline & Derechos & \\
\hline
\end{tabular}

\section{Resultados}

En el estudio sobre la formación de usuarios en las bibliotecas universitarias españolas, antes comentado, se contabilizaron un total de quince materiales formativos en el año 2006. El primer dato que podemos aportar comparando la situación actual con la de entonces es el incremento del número de recursos, que han pasado de ser 15 a los actuales 72 .

Otro dato significativo a resaltar se refiere a la creación de los recursos por parte de la biblioteca o a la utilización de material formativo de otros centros. Los 72 recursos analizados pertenecen sólo a 17 centros (24\%), mientras que los cincuenta y cinco restantes (76\%) utilizan guías de consulta o los enlaces a otros recursos externos como soporte a la formación. Entre los tutoriales externos más enlazados desde las páginas web de las bibliotecas se encuentran TILT (Texas Information Literacy Tutorial) de la University of Texas System Digital Library; InfoSuss Information Literacy Tutorial de la University de Sussex; InfoSphère de l'Université de Montreal, e INTUTE: virtual training suite de la JISC. En el caso de los españoles hay muchos enlaces a los manuales en español de los diferentes distribuidores de bases de datos y de los gestores bibliográficos, RefWorks principalmente. La guía Com elaborar un treball acadèmic de la Biblioteca de la Pompeu Fabra también está enlazada desde diversas páginas. 
Las adaptaciones y traducciones también son frecuentes. Así, TILT (Texas Information Literacy Tutorial) de la Universitat Politècnica de Catalunya es la adaptación del tutorial con el mismo nombre de la University of Texas System Digital Library; el Tutorial en cerca d'informació del CRAI de la Universitat de Barcelona es una adaptación de LOBO (Library Online Basic Orientation) de la North Carolina State University Libraries y Búsqueda de información de la Biblioteca de la Universidad de Sevilla es una adaptación de Library Research Tutor de la University of California San Diego, que ha dejado de ser consultable recientemente.

\subsection{Aspectos generales}

Respecto a los idiomas, sesenta y cinco presentan los contenidos en castellano $(90 \%)$, seguido del catalán con cuatro (6\%) y el gallego con tres (4\%). No existe en la actualidad ningún material formativo traducido a varios idiomas.

Un poco menos de la mitad de los materiales, concretamente veintisiete, incluyen la fecha de creación (37,5\%) siendo ésta inferior a los cinco años. Este dato indica que se trata de recursos recientes y con poca trayectoria aún en nuestras bibliotecas.

Más significativa es la fecha de actualización, ya que en este caso sólo dos materiales $(2,78 \%)$ cuentan con esta información.

En cuanto a la presencia de elementos informativos de contexto (es decir, la explicación de la historia y del proceso de creación del recurso), destacar que es un indicador inexistente en el 98,7\% de los materiales y que tan sólo uno, la adaptación del tutorial TILT de la UPC, lo incluye.

En lo que respecta a la inclusión de información sobre los responsables que han intervenido en la creación del material formativo, son tan sólo tres recursos los que contienen indicación de autoría (4\%).

\subsection{Organización y tipología de los contenidos}

Los contenidos son muy diversos y, en la mayoría de los casos, hacen referencia a un recurso concreto o a servicios de la biblioteca.

En cuanto a la producción se tiene que destacar que siete centros han elaborado un único recurso propio, disponiendo los otros diez de más de uno.

Sobre la extensión de los contenidos, es importante señalar que existen dieciséis materiales $(22,22 \%)$ que han sido diseñados para ser impresos puesto que están en formato $P D F$. El resto, cincuenta y seis, pueden ser visualizados y leídos por pantalla $(77,78 \%)$.

Respecto a la estructura se puede destacar que tan sólo ocho recursos formativos (11\%) organizan sus contenidos en módulos, siendo los bloques de tres módulos (tres materiales), y de cuatro módulos (dos materiales) los más utiliza- 
dos. La estructura modular es una solución idónea para contenidos exhaustivos o para organizar una diversidad de contenidos.

En lo que respecta a la tipología de los contenidos comentar que en la mayoría encontramos texto combinado con imágenes que pueden ser fotografías, iconos para ilustrar el contenido o gráficos. El predominio es el de la combinación de texto con las pantallas de los recursos a explicar $(61,11 \%)$, seguido de lejos por fotografías e iconos $(8,33 \%)$.

Los materiales elaborados por las bibliotecas universitarias españoles están muy relacionados con recursos concretos o la difusión de sus servicios, es decir, que tienen un carácter muy instrumental. En general, se centran en contenidos sobre recursos temáticos por especialidades (15,28\%) y en el catálogo $(9,72 \%)$. Señalar que sólo hay tres materiales formativos $(4,17 \%)$ que tratan sobre alfabetización informacional, es decir, que enseñan a evaluar las necesidades de información, a conocer las diferencias entre las fuentes, a buscar en ellas y a evaluar y comunicar la información de manera eficaz.

\subsection{Aspectos docentes}

La mayor parte de los materiales incluyen ejemplos prácticos para ilustrar los contenidos explicados. Concretamente sesenta y tres (87,5\%) implementan algún caso práctico ejemplificador.

Sólo un material contiene un glosario, la adaptación de TILT.

En el caso de los enlaces externos encontramos que veintidós materiales (31\%) contienen enlaces.

La metodología docente empleada por los materiales presenta pocas variaciones. La expositiva, con diferentes ejemplos, es la que mayoritariamente predomina $(56,94 \%)$, la demostración guiada es la segunda opción más utilizada (40,28\%), a partir de una búsqueda se enseña de manera pautada cada uno de los pasos de un entorno o recurso concreto, y la expositiva con ejercicios (2,78\%) es la tercera opción aunque muy alejada de las anteriores. No hemos encontrado otras combinaciones ni elementos más innovadores como prácticas o la metodología de la resolución de problemas, aprendizaje por descubrimiento o aprendizaje colaborativo.

\subsection{Evaluación del alumno}

La incorporación de ejercicios es básica y se trata del recurso docente de evaluación por excelencia. Los ejercicios permiten reforzar conceptos o procedimientos que han sido aprendidos a lo largo del tutorial. Es muy significativo que tan sólo seis materiales formativos contienen ejercicios (8\%) siendo el cuestionario el tipo de ejercicio utilizado por todos de ellos, aunque la adaptación de TILT, realiza este cuestionario en forma de juegos. Todos los materiales emplean formas 
de retroalimentación a la hora de contestar a las preguntas de los alumnos y en cinco de ellos se puntúan y evalúan los ejercicios.

\subsection{Asistencia}

En este punto se identifican los recursos ofrecidos por el material para que el alumno contacte con el responsable de la formación, le plantee sus dudas o le resuelva problemas relacionados con el mismo. Esta posibilidad sólo está presente en treinta y cinco de ellos $(48,61 \%)$, siendo el tipo de asistencia el correo electrónico (44\%), el formulario (2,78\%) o el correo electrónico y teléfono $(1,39 \%)$.

\subsection{Evaluación del recurso formativo}

Este apartado identifica la posibilidad de que el material pueda ser evaluado por el alumno, aquí se debería incluir la posibilidad de evaluar lo que se ha aprendido, poder opinar sobre el proceso de aprendizaje y las cuestiones relativas a los aspectos formales del mismo. Se trata de un elemento clave para detectar errores e implementar mejoras en todos los sentidos. Sorprende que sólo uno, TILT (1\%), incluya un sistema de evaluación.

\subsection{Elementos que favorecen la autonomía del proceso de aprendizaje}

Sólo seis materiales (8\%) contienen objetivos formativos que no se limitan a una presentación general de los contenidos, mientras que los sesenta y seis restantes (92\%) no incluyen esta información qué creemos fundamental.

No todos los alumnos poseen los mismos conocimientos y habilidades sobre una materia. Por tanto, sería conveniente marcar diferentes rutas de aprendizaje en función de lo que se sabe a priori sin presuponer que todos los alumnos saben lo mismo. Para ello suele ser recomendable incorporar un sistema previo, que indica qué sabe el alumno y a partir de qué punto concreto tiene que seguir con el tutorial. En la mayoría de los casos este control suele hacerse en forma de cuestionario. En el caso de los materiales españoles ninguno incorpora un sistema o pretest para evaluar los conocimientos previos.

Otro elemento muy útil para favorecer la autonomía y planificación del proceso de aprendizaje del alumno es el de indicar el tiempo que empleará en la ejecución de todo o de parte, en el caso de que el material disponga de una organización modular. En el caso de los materiales españoles encontramos que sólo diez contienen esa información (14\%). 
La sumarización es un elemento didáctico que permite conocer de manera sintetizada los conceptos o habilidades que se acaban de aprender y, en algunos casos, adelanta los que quedan por conocer. Esta utilidad sólo se ha incorporado en tres de los materiales formativos analizados (4\%).

\subsection{Elementos que contemplan alumnos con conocimientos diferentes}

No existe ningún material español que incorpore diferentes niveles de profundidad de los contenidos según los conocimientos del alumno. No todos los alumnos saben lo mismo, por lo que sería recomendable un nivel de contenidos diferentes. Si creamos un recurso formativo básico será imposible que los usuarios con más conocimientos puedan avanzar y profundizar.

Más dificultades plantea el determinar la audiencia del material, es decir, si éste está destinado a una tipología concreta de usuario. Sólo se ha podido determinar claramente en dos casos, uno porque incorpora una parte común y el resto para cada perfil de usuario y otro, que genera un recurso docente diferenciado para cada tipología de usuario.

Otro elemento a destacar es que ninguno de los materiales estudiados contiene un módulo para la educación a distancia, como tampoco existe ninguno que se dedique íntegramente a ella.

\subsection{Navegabilidad, usabilidad y accesibilidad}

Sólo un material (1,39\%) incorpora información adicional de cómo moverse o navegar por sus contenidos.

Sólo dos contienen mapa del contenido o site map (3\%). Este elemento permite que el usuario vea de manera resumida todos los contenidos detallados y pueda acceder a ellos de manera directa sin tener que hacer una consulta lineal. En los dos casos se trata de mapas de contenido textuales.

Otro elemento que sitúa al alumno y le orienta respecto a la navegación es el de la barra de situación. Sólo uno de los materiales analizados lo contiene.

En cuanto a la estructura de los contenidos en pantalla vemos que la gran mayoría, cincuenta y nueve (82\%), lo hace a partir de un área donde se ubican los contenidos, el 17\% lo hace en dos áreas y un 1\% utiliza tres.

No existe ninguna forma de personalización de colores o del aspecto del recurso.

No se ha encontrado ningún elemento que favorezca la accesibilidad como se recomienda en el caso de las páginas web. No hay opción de cambiar el tamaño de la letra o el contraste de las pantallas

La interactividad de la interfaz del material se consigue con el soporte tecnológico adecuado, pero sólo el 19,44\% de los materiales contienen una interfaz 
interactiva (todos ellos realizan la consulta desde Macromedia Flash, y dos de ellos combinan HTML con SWF el formato de Flash).

La inclusión de elementos de sonido es también baja ya que el audio está presente solamente en quince de los materiales (21\%). En dos de ellos, los contenidos pueden ser escuchados íntegramente, y en el resto contienen elementos de audio para favorecer aspectos de la interactividad del sistema o se incorpora música para completar el recurso docente.

Para finalizar, comentar que ninguno de los setenta y dos materiales presenta contextos didácticos, es decir escenarios concretos que hacen que el recurso sea más atractivo al alumno y por tanto, favorezca la interactividad con el sistema. Generalmente suelen presentar la información de una manera menos académica y más divertida para facilitar la interacción y motivación del alumno.

\subsection{Características tecnológicas}

Ninguno de los materiales consultados guarda el perfil de usuario mediante un código de entrada.

En referencia al formato o soporte tecnológico de los materiales formativos (Tabla II) sorprende encontrar que el principal sea el PPS $(48,61 \%)$ de PowerPoint más propio para presentaciones orales, seguido del formato PDF de Adobe Acrobat (20,83\%), un formato preparado para la impresión en papel y nada idóneo para facilitar ningún tipo de animación ni interactividad. La tercera opción son los recursos que tienen que ser consultados desde Macromedia Flash $(19,44 \%)$.

TABLA II

Principales formatos de los materiales formativos

\begin{tabular}{l|c|c}
\hline \multicolumn{1}{c|}{ Formatos } & Total & \% \\
\hline Pps & 35 & 48,61 \\
\hline Pdf & 15 & 20,83 \\
\hline SWF & 14 & 19,44 \\
\hline Html & 5 & 6,94 \\
\hline Html y SWF & 2 & 2,78 \\
\hline
\end{tabular}

En cuanto a la descarga de plugins para facilitar la visualización en el caso de que el usuario final no disponga del programa, destacar que sólo uno permite la descarga de plugins Búsqueda de información (1\%).

No hemos encontrado ningún material que facilite una versión textual del contenido del recurso docente de manera adicional. 


\section{Conclusiones}

Este análisis de los materiales formativos efectuado dos años después de la realización de la encuesta, nos lleva a afirmar que existen pocos elementos de la ALFIN que se hayan asumido en este momento. No se observan muchas mejoras en este campo a pesar de que las bibliotecas vieran el desarrollo de los recursos formativos como un objetivo a conseguir. Si bien es cierto que el número de materiales se ha incrementado desde la encuesta, desde quince títulos en 2006 a setenta y dos en 2008, lo más importante a destacar es que las bibliotecas denominan tutorial a un material que no lo es. Si aplicamos los dos criterios que consideramos fundamentales, como son que incorporen objetivos formativos y algún tipo de ejercicio, sólo cinco de los materiales analizados cumplirían ambos criterios. Otro elemento clave es el de la temática de los materiales formativos. En la actualidad poco ha cambiado respecto a la formación tradicional, los contenidos continúan centrados en la formación de carácter instrumental que explica los recursos y su funcionamiento. Sólo hay tres materiales que consideraríamos de alfabetización informacional y además dos son una adaptación de recursos internacionales TILT (Texas Information Literacy Tutorial) y LOBO ( $\mathrm{Li}$ brary Online Basic Orientation).

El desarrollo y creación de estos materiales están concentrados en pocas instituciones, sólo diecisiete centros que corresponde al 24\% de las bibliotecas universitarias españolas han creado un recurso propio, mientras que el $76 \%$ de las bibliotecas utilizan materiales externos, situación que esperamos pueda mejorar en un futuro próximo. A nivel tecnológico hay pocos elementos de cambio desde la descripción del 2006, el formato más utilizado en la elaboración de materiales sigue siendo el PPS de PowerPoint (48,61\%) y el PDF de Adobe (20,83\%), desaparecen los formatos para ser tratados por los procesadores y aparece $S W F$ de Flash (19,44\%). Este dato indica que los materiales son poco interactivos ya que la tecnología empleada es más idónea para las presentaciones orales y la impresión de la información. Otro aspecto a tener en cuenta es el de la metodología docente con la que se ha diseñado y pensado el material formativo. Aunque según los datos de la encuesta se aplicaban metodologías innovadoras, como el aprendizaje a partir de la resolución de problemas (43,75\%), no se refleja en el diseño, puesto que no hay ningún material que siga el modelo constructivista. La expositiva con ejemplos es la primera, con un 56,94\%, seguida de las demostraciones guiadas, con un 40,28\%. Si bien es cierto que la temática puede determinar la elección de una metodología concreta, en el caso de las bases de datos o del catálogo resulta muy útil enseñar la interfaz a partir de demostraciones guiadas, la expositiva implica una menor complejidad en el diseño didáctico del material.

Es evidente que las bibliotecas españolas han expresado su voluntad y sus esfuerzos para favorecer la creación y organización de los materiales formativos. Este interés se manifiesta en dos líneas de actuación concretas: el desarrollo de materiales para la formación y la creación de depósitos institucionales de materiales didácticos y objetos de aprendizaje tal como se indica en el II Plan Estra- 
tégico 2007-2010 de REBIUN. Como se ha descrito la calidad actual de los materiales formativos de las universidades españolas es bastante deficitaria y no se adaptan aún al contexto de la ALFIN. La explicación cabe buscarla en algunos datos extraídos de la encuesta que indican que los materiales formativos están elaborados por el personal de biblioteca, el mismo que se encarga de impartir la docencia. Estos formadores son poco competentes en el ámbito de la didáctica y de la tecnología ya que el criterio prioritario para su selección son los conocimientos sobre la materia. Las bibliotecas no son conscientes de estas necesidades puesto que los docentes reciben la misma formación que el resto del personal. Esta situación sumada a la falta de colaboración con otros departamentos o servicios de las universidades como los informáticos y pedagógicos da como resultado unos materiales formativos poco efectivos desde el punto de vista docente y poco interactivos desde el tecnológico. La transformación de las bibliotecas universitarias al modelo CRAI podría subsanar estas deficiencias.

Como idea general destacar el hecho de que los materiales formativos de las bibliotecas españolas se encuentran en un estadio inicial y muchos son los elementos que deben ser mejorados con urgencia, insistiendo principalmente en dos básicos: la incorporación de objetivos formativos y de ejercicios que refuercen lo aprendido. Otro elemento que no conlleva un coste elevado es la incorporación de algún sistema de evaluación del recurso docente, ya que sin este instrumento no se puede saber, finalmente, si el material formativo ha cumplido su cometido o no y si han valido la pena los esfuerzos y los recursos invertidos en su elaboración.

En cuanto a las mejoras didácticas sería muy recomendable incorporar diferentes niveles de contenidos teniendo en cuenta que no todos los alumnos saben lo mismo, esto se debería completar con alguna forma de evaluación previa como un pretest. Mejoras didácticas menos costosas serían la incorporación de un glosario que asegure la comprensión de la terminología más especializada, y también de opciones que favorecen la autonomía del alumno como la temporalización de los módulos o del recurso docente íntegro y la sumarización de los contenidos.

Otros elementos, quizá no tan fundamentales, irían en la línea de completar más la información relativa a las ayudas y la incorporación de los responsables de la formación para contactar con ellos en caso de problemas o sugerencias. Complementado lo anterior se deberían incorporar elementos que doten de transparencia al tutorial, como por ejemplo las personas que han intervenido en el proceso de creación e indicar, como mínimo, la fecha de la última actualización de los contenidos, de la misma manera que se exige este criterio a la hora de evaluar los contenidos de las páginas web.

\section{Bibliografía}

Campal, M. Felicidad; y Domínguez Sanjurjo, Ramona (2006): La formación de usuarios y alfabetización informacional en la BPE de Salamanca "Casa de las Conchas". Educación y Biblioteca, $\mathrm{n}^{\circ}$ 156. pp. 112-117. 
Gómez Enrich, Roser, et al. (2002): AABIB: La autoformación de usuarios en las Bibliotecas de la UPC a través de Internet. XII Jornadas Bibliotecarias de Andalucía. Sevilla: $\mathrm{AAB}$.

Martín, Wigberta, y Moscoso, Purificación (1994): Conocer el MESH: un programa de instrucción asistida por ordenador para el uso del Medical Subject Headings. 4as Jornadas Españolas de Documentación Automatizada. Gijón: FESABID.

Marzal García-Quismondo, Miguel Ángel; Calzada Prado, Javier, y Vianello, Marina (2008): Criterios para la evaluación de la usabilidad de los recursos educativos virtuales: un análisis desde la alfabetización en información. Information Research, vol. 13, $\mathrm{n}^{\circ} 4$.

Méndez, Montserrat, et al. (2002): FIBU: Formación Inteligente en las Bibliotecas de la UPC. III Jornadas de Bibliotecas Digitales: (JBIDI'O2). El Escorial (Madrid): JBIDI.

Nielsen, Jacob (2000): Usabilidad: diseño de sitios web. Madrid [etc.]: Prentice Hall.

Nielsen, Jacob, y Loranger, Hoa (2006): Usabilidad: prioridad en el diseño web. Madrid: Anaya Multimedia.

Parcerisa Aran, Artur (2001): Materiales curriculares: cómo elaborarlos, seleccionarlos y usarlos. Barcelona: Graó.

Richadeau, François (1986): Conception et production des manuels scolaires: guide pratique. Paris: UNESCO.

Ribes Llopis, Inmaculada (1996): SIRIO: Tutorial multimedia sobre sistemas de recuperación de la Información. 5as Jornadas Españolas de Documentación Automatizada. Cáceres: FESABID.

Sastre Miralles, Natalia (2000): Productos y servicios para la formación de usuarios de bibliotecas universitarias: el uso de bases de datos en entornos Web. Tas Jornadas Españolas de Documentación Automatizada. Bilbao: FESABID.

Seguí, Rosa; Bosch Pou (2001): Productos multimedia aplicados al aprendizaje de las técnicas de recuperación en bases de datos: Sirio y Hot Copy Searching Dialog Tutorial. Actas del V Congreso ISKO-España. Alcalá de Henares: ISKO; Universidad de Alcalá.

Somoza-Fernández, Marta (2002): Avaluació dels tutorials de PubMed i Eric. BiD: textos universitaris de Biblioteconomia i Documentació, vol. 9. http://www.ub.es/biblio/ bid/09somoza.htm. [Consulta 13 febrero 2009]

Somoza-Fernández, Marta (2005): Eines per a la creació d'exercicis al Web. BiD: textos universitaris de Biblioteconomia i Documentació, num. 14. http://www2.ub.es/bid/ consulta articulos.php?fichero=14somoz2.htm. [Consulta 13 febrero 2009]

Somoza-Fernández, Marta, y Abadal, Ernest (2007): La formación de usuarios en las Bibliotecas universitarias españolas. El Profesional de la información, vol. 16, $\mathrm{n}^{\circ} 4$, pp. 287-293.

\section{Anexo. Relación de los materiales formativos españoles analizados}

El análisis se realizó entre julio y agosto de 2008. Los enlaces han sido revisados en junio de 2009, en aquellos casos en los que no consta la URL se debe a que ya no se encuentran accesibles en la actualidad. 
Bases de datos en Ciencias Sociales

Universidad de Jaén

URL: http://www.ujaen.es/serv/biblio/formacion/bdsociales.pdf

\section{Bases de datos en Filología}

Universidad de Jaén

URL: http://www.ujaen.es/serv/biblio/formacion/bdfilologias.pdf

Bases de datos en Psicología

Universidad de Jaén

\section{Biblioteca digital en Ciencias de la Salud}

Universidad de Jaén

URL: http://www.ujaen.es/serv/biblio/formacion/bca\%20digital ccss.pdf

\section{Boletines de sumarios}

Universidad de Jaén

URL: http://www.ujaen.es/serv/biblio/formacion/bolsumarios.pdf

\section{Bucear y encontrar en Internet}

Universidad de Jaén

URL: http://issuu.com/biblioteca universitaria jaen/docs/bucear internet

\section{Búsqueda de información}

Biblioteca Universidad de Sevilla

URL: http://librisql.us.es/ximdex/flash/index.html

\section{Cómo buscar en la base de datos Cuiden}

Universidad de Jaén Temática: Bases de datos

URL: http://issuu.com/biblioteca universitaria jaen/docs/cuiden09

\section{Cómo citar en el área de Ciencias de la Salud}

Universidad de Jaén

URL: http://www.ujaen.es/serv/biblio/formacion/como citar.pdf

Cómo elaborar un trabajo académico

Biblioteca de la Universidad Complutense de Madrid

URL: http://www.ucm.es/BUCM/alfin/21087.php

\section{Cómo elaborar un trabajo científico}

Universidad de Jaén

\section{Cómo elaborar una bibliografía}

Biblioteca y Archivo de la Universidad Autónoma de Madrid

URL: http://biblioteca.uam.es/sc/guiasytutoriales.html

\section{Cómo hacer una reserva en Fama}

Biblioteca Universidad de Sevilla

URL: http://bib.us.es/aprendizaje investigacion/guias tutoriales/common/tutoriales/reservasFama.swf 
Cómo usar el Préstamo Interbibliotecario

Biblioteca de la Universidad de la Laguna

URL: http://www.bbtk.ull.es/Private/folder/Servicios/Formacion/Tutoriales/ pi.pps

\section{Consulta bibliográfica}

Biblioteca Universidad de Valladolid

URL: http://biblioteca.uva.es/biblioteca/ayudas/busquedas/busquedas.htm

\section{Consulta y uso de PubMed}

Biblioteca Universidad de Valladolid

URL: http://biblioteca.uva.es/biblioteca/ayudas/PubMed1024/PubMed1024. $\underline{\mathrm{htm}}$

\section{Dialnet: tutorial}

Biblioteca y Archivo de la Universidad Autónoma de Madrid

URL: http://biblioteca.uam.es/sc/guiasytutoriales.html

\section{Estrategias de búsqueda}

Universidad de Jaén

URL: http://www.ujaen.es/serv/biblio/formacion/estrategias.pdf

\section{Harrison Online en español}

Biblioteca de la Universidad Complutense de Madrid

URL: http://www.ucm.es/BUCM/med/doc7922.swf

\section{La documentación en Ciencias Sociales}

Universidad de Jaén

URL: http://www.ujaen.es/serv/biblio/formacion/docuccss.pdf

\section{Las revistas en el catálogo, paso a paso}

Biblioteca y Archivo de la Universidad Autónoma de Madrid

URL: http://biblioteca.uam.es/sc/guiasytutoriales.html

\section{OPAC básico}

Biblioteca de la Universidad de la Laguna

URL: $<$ http://www.bbtk.ull.es/Private/folder/Servicios/Formacion/Tutoriales/ opacbasicoAbsysNet.pps

\section{OPAC búsquedas avanzadas}

Biblioteca de la Universidad de la Laguna

\section{Plataforma PROQUEST}

Universidad de Jaén

URL: http://www.ujaen.es/serv/biblio/formacion/proquest.pdf

\section{Préstamo a domicilio}

Universidad de Jaén

URL: http://www.ujaen.es/serv/biblio/formacion/prestamo.pdf 


\section{Recursos electrónicos y Bibliotecas digitales}

Universidad de Jaén

URL: http://www.ujaen.es/serv/biblio/formacion/recurweb.pdf

\section{RefWorks}

Biblioteca Universidad Carlos III

URL: http://www.uc3m.es/portal/page/portal/biblioteca/aprende usar/ tutoriales y ayudas/curso-sobre-refworks.pdf

\section{Revistas electrónicas en Ciencias Sociales}

Universidad de Jaén

URL: http://www.ujaen.es/serv/biblio/formacion/e-revistassociales.pdf

\section{Servicio al usuario: PIN}

Biblioteca Universidad de Valladolid

URL: http://biblioteca.uva.es/biblioteca/Ayudas/pin/pin.htm

\section{Servicios de la Biblioteca}

Universidad de Jaén

URL: http://www.ujaen.es/serv/biblio/formacion/servicios buja.pdf

\section{Sumarios de revistas}

Biblioteca Universidad de Valladolid

URL: http://biblioteca.uva.es/biblioteca/ayudas/busquedas/busquedas.htm

\section{TILT (Texas Information Literacy Tutorial)}

Servei de Biblioteques i Documentació de la Universitat Politècnica de Catalunya

URL: http://bibliotecnica.upc.es/bib160/tilt/

\section{Titorial CRAI}

Biblioteca Universidade de Santiago de Compostela

URL: http://bibliotecnica.upc.es/rebiun/nova/jornadas/CRAI/videocrai2.html

\section{Titorial RefWorks: opcións avanzadas}

Biblioteca Universidade de Santiago de Compostela

URL: http://busc.usc.es/Servizos/Formacion/RefWorksOpcionsAvanzadas.ppt

\section{Titorial RefWorks: opcións básicas}

Biblioteca Universidade de Santiago de Compostela

URL: http://busc.usc.es/Servizos/Formacion/RefwoksOpcionsBasicas.ppt

\section{Tutorial básico para el uso de Papyrus}

Biblioteca Universidad de Oviedo

URL: http://buo.uniovi.es/tutoriales/Papyrus10.htm

\section{Tutorial búsqueda rápida en el catálogo}

Biblioteca Universidad de la Rioja

URL: http://biblioteca.unirioja.es/tutoriales/busrapida/busquedarapida.html 


\section{Tutorial de Biblioteconomía}

Biblioteca Universidad Carlos III

URL: http://www.uc3m.es/portal/page/portal/biblioteca/aprende usar/ tutoriales_y_ayudas/biblioteconomia.pdf

\section{Tutorial de Derecho}

Biblioteca Universidad Carlos III

URL: http://www.uc3m.es/portal/page/portal/biblioteca/aprende usar/ tutoriales y ayudas/fuentes-derecho-informaci $\% \mathrm{~F} 3$ n-electr $\% \mathrm{~F} 3$ nica.pdf

\section{Tutorial de Economía}

Biblioteca Universidad Carlos III

URL: http://www.uc3m.es/portal/page/portal/biblioteca/aprende usar/ tutoriales y ayudas/ECONOMIA-2008.pdf

\section{Tutorial de Estadísticas}

Biblioteca Universidad Carlos III

URL: http://www.uc3m.es/portal/page/portal/biblioteca/aprende usar/ tutoriales y ayudas/fuentes\%20estadisticas.pdf

\section{Tutorial de Ingeniería}

Biblioteca Universidad Carlos III

URL: http://www.uc3m.es/portal/page/portal/biblioteca/aprende usar/ tutoriales y ayudas/Fuentes\%20en\%20Ingenieria.pdf

\section{Tutorial de la Biblioteca de Medicina}

Biblioteca Universitat Autònoma de Barcelona

URL: http://clon.uab.es/tutorialBM/

\section{Tutorial de las bases de datos de ProQuest}

Biblioteca de la Universidad Complutense de Madrid

URL: http://www.ucm.es/BUCM/tutoriales/proquest/

\section{Tutorial de Legislación y Jurisprudencia}

Biblioteca Universidad Carlos III

URL: http://www.uc3m.es/portal/page/portal/biblioteca/aprende usar/ tutoriales_y_ayudas/tutorial-legis-juris.pdf

\section{Tutorial de Patentes (1)}

Biblioteca Universidad Carlos III

URL: http://www.uc3m.es/portal/page/portal/biblioteca/aprende_usar/ tutoriales y ayudas/Patentes\%20I.pdf

\section{Tutorial de Patentes (2)}

Biblioteca Universidad Carlos III

URL: http://www.uc3m.es/portal/page/portal/biblioteca/aprende usar/ tutoriales y ayudas/Patentes\%20II.pdf 
Tutorial de Política y Sociología

Biblioteca Universidad Carlos III

URL: http://www.uc3m.es/portal/page/portal/biblioteca/aprende_usar/ tutoriales y ayudas/cps.pdf

Tutorial de recursos electrónicos de la Red de bibliotecas del CSIC

Red de Bibliotecas del CSIC

URL: http://www.csic.es/cbic/formacion/eerecursos.htm

\section{Tutorial de revistas electrónicas}

Biblioteca Universidad Carlos III

URL: http://www.uc3m.es/portal/page/portal/biblioteca/aprende_usar/ tutoriales y ayudas/tutorial-revistas-electronicas.pdf

\section{Tutorial de servicios de Biblioteca}

Biblioteca Universidad de Extremadura

URL: http://biblioteca.unex.es/guia/tutorial.pps

\section{Tutorial de SFX}

Biblioteca y Archivo de la Universidad Autónoma de Madrid

URL: http://biblioteca.uam.es/sc/guiasytutoriales.html

\section{Tutorial de Turismo}

Biblioteca Universidad Carlos III

URL: http://www.uc3m.es/portal/page/portal/biblioteca/aprende usar/ tutoriales y ayudas/tutorial-turismo.pdf

\section{Tutorial de la Unión Europea}

Biblioteca Universidad Carlos III

URL: http://www.uc3m.es/portal/page/portal/biblioteca/aprende usar/ tutoriales_y_ayudas/tutorial-union-europea.pdf

\section{Tutorial de uso del Catálogo de la Biblioteca Universitaria}

Biblioteca de la Universidad de las Palmas de Gran Canaria

URL: http://biblioteca.ulpgc.es/files/repositorio de docum152/guias/catalogo/ tutorial catalogo/Como\%20utilizar\%20el\%20catalogo.pps

\section{Tutorial del catálogo}

Biblioteca y Archivo de la Universidad Autónoma de Madrid

URL: http://biblioteca.uam.es/sc/guiasytutoriales.html

\section{Tutorial en cerca d'informació}

CRAI Universitat de Barcelona

URL: http://www.bib.ub.edu/atri/tutorial1/

\section{Tutorial i.Xpertutor}

Servei de Biblioteca i Documentació de la Universitat Rovira i Virgili URL: http://www.urv.cat/man/tutorial/ 
Tutorial OPAC: Catálogo de la biblioteca

Biblioteca Universidad Carlos III

URL: http://www.uc3m.es/portal/page/portal/biblioteca/aprende usar/ tutoriales y ayudas/tutorial-opac.pdf

Tutorial para la Solicitud de documentos a bibliotecas de la Red del CSIC y a bibliotecas externas

Red de Bibliotecas del CSIC

URL: http://www.csic.es/cbic/documents/solicitud documentos usuarios csic. ppt

Tutorial para la utilización y consulta de JCR

Biblioteca Universidad de Oviedo

URL: http://www.uniovi.es/zope/Biblioteca/ibipi/indices de impacto/jcr tuto$\underline{\text { rial/ }}$

Tutorial por perfil usuario: docentes e investigadores

Biblioteca y archivo de la Universidad Autónoma de Madrid

URL: http://biblioteca.uam.es/sc/guiasytutoriales.html

Tutorial por perfil usuario: estudiantes

Biblioteca y archivo de la Universidad Autónoma de Madrid

URL: http://biblioteca.uam.es/sc/guiasytutoriales.html

\section{Tutorial por perfil usuario: PAS}

Biblioteca y archivo de la Universidad Autónoma de Madrid

URL: http://biblioteca.uam.es/sc/guiasytutoriales.html

\section{Tutorial por perfil usuario: visitantes}

Biblioteca y archivo de la Universidad Autónoma de Madrid

URL: http://biblioteca.uam.es/sc/guiasytutoriales.html

\section{Tutorial revistas electrónicas}

Biblioteca Universidad de Valladolid

URL: http://cigales.cpd.uva.es/biblioteca/ayudas/RevElec/RevElec.htm

Tutorial sobre el uso de digital. CSIC

Red de Bibliotecas del CSIC

URL: http://digital.csic.es/info/demo/demo.htm

Tutorial sobre mi cuenta Biblioteca Universidad de Sevilla

URL: http://bib.us.es/aprendizaje investigacion/guias tutoriales/common/tutoriales/micuentadefin.swf

Tutorial sobre Peticiones de fotocopias y préstamos a las bibliotecas de la Red de Bibliotecas del CSIC

Red de Bibliotecas del CSIC

URL: http://www.csic.es/cbic/documents/solicitud documentos usuarios externos_csic_sin_bibliotecas.ppt 


\section{Tutorial Web of Knowledge-Current Contents}

Biblioteca Universidad Carlos III

URL: http://www.uc3m.es/portal/page/portal/biblioteca/aprende usar/ tutoriales y ayudas/curso-sobre-WOK-CCC.pdf

\section{Westlaw España}

Biblioteca y Archivo de la Universidad Autónoma de Madrid

URL: http://biblioteca.uam.es/sc/guiasytutoriales.html

\section{¿Qué es la alfabetización informacional?}

Biblioteca Universidad de Sevilla

URL: http://bib.us.es/aprendizaje investigacion/guias tutoriales/common/INFLIT.ppt. 